\section{Diagnostic tools for female urethral diverticulum: Current perspectives}

\author{
Gloria Calagna, ${ }^{1}$ Marco Vella, ${ }^{2}$ \\ Maria Elena Mugavero, ${ }^{1}$ \\ Giorgio Gugliotta, ${ }^{3}$ Salvatore Polito, ${ }^{4}$ \\ Antonino Perino, ${ }^{1}$ Gaspare Cucinella ${ }^{1}$
}

'Obstetrics and Gynecology Unit, "Villa Sofia Cervello" Hospital, University of Palermo; ${ }^{2}$ Surgical Disciplines, Oncology and Dentistry, University of Palermo, Palermo; ${ }^{3}$ Urogynecology Unit, "Villa Sofia Cervello" Hospital, Palermo; ${ }^{4}$ Obstetrics and Gynecology Unit, “G. Martino" Hospital, University of Messina, Italy

\begin{abstract}
Although once considered quite a rare condition in the past, female urethral diverticulum (UD) would now appear to have a higher frequency, perhaps due to greater attention from physicians. To date, there is no agreement on which is the best method for diagnosis of female UD. Traditionally, the approach was based on quite invasive techniques, such as voiding cystourethrography, and double-balloon urethrography, with satisfactory results but relevant limitations. More recent high-resolution imaging techniques, such as 2D-3D ultrasonography (US) and magnetic resonance imaging (MRI) have also been applied in the study of the abnormalities of the female urethra. US had the advantage of the outpatient setting, non-invasiveness and absence of contrast medium use; MRI, is characterized by high sensitivity thanks to multiplanar capability, with an optimal characterization of periurethral diseases or its abnormalities, and lack of ionizing radiation. A real innovation is represented by computer tomography-voiding cystourethrography, a rapid technique that allows for high quality simultaneous 2D and 3D images of the urethra, well correlated to MRI and consequently with surgical results. Here, we report and comment the available tools in the diagnosis of female UD, focusing particularly on pros and contra of different methods.
\end{abstract}

\section{Introduction}

Female urethral diverticulum (UD) was first described in medical literature in 1805 by William Hay. ${ }^{1}$ It is defined as a localized sac-like herniation, continuous with the ure- thral lumen, between the periurethral fibromuscular fascia and anterior vaginal wall. ${ }^{2}$ A population-based analysis in Olmsted County (USA) reported a low incidence affecting fewer than 20 women out of $1,000,000$, with an incidence of $0.02 \%$ per year. ${ }^{3}$ Although considered quite a rare condition in the past, female UD seems to have much more frequent occurrence today, perhaps due to greater attention from physicians, with a current prevalence estimated between 0.6 and $6.0 \%{ }^{4-6}$

The etiology of acquired UD is still to define. In most cases, a congenital origin is attributed and major incidence is in the third to fifth decade. ${ }^{6,7}$ The majority of UD are located in the middle third of the urethra and involve the postero-lateral wall, resulting from enlargement of obstructed periurethral glands. ${ }^{8} 9$ Periurethral glands are located in the distal two-thirds of the female urethra terminating in the paraurethral glands of Skene (variable in number from 6 to 30 ): all paraurethral ducts empty secretions into the urethral lumen. Possible causal factors of UD formation include urethral injury during childbirth, previous surgery and repetitive trauma. ${ }^{10}$

Approximately $20 \%$ of patients with UD are asymptomatic and the symptoms, when present, do not seem to be related to the size or number of diverticula. ${ }^{11}$ UD may be small $(4-5 \mathrm{~mm})$ or large (until $3 \mathrm{~cm}$ or more), single or multiple, round shaped or circumferentially or horseshoe shaped. Dysuria, post-void dribbling, dyspareunia, recurrent urinary tract infections, periurethral mass on physical examination are the most common symptoms and findings of UD.

Complications of UD can occur, including abscess, intra-diverticular calculus and, less commonly, neoplasm. ${ }^{7}$

For these reasons, UD diagnosis is often not easy, and it is estimated that the mean time from onset of symptoms and UD diagnosis is about 24 months. ${ }^{12}$ Thus, the most important factor for UD diagnosis is to actually think of it and, consequently, look for it. While clinical history and physical examination are the first diagnostic steps, imaging is at the same time important to confirm the findings of clinical evaluation or to find UD without clinical evidence. ${ }^{13}$

To date, there is no agreement on which the best diagnostic method is for female UD. The older, traditional and more invasive techniques used are voiding cystourethrography (VCUG), double-balloon urethrography (DBU) and urethro-cystoscopy (UC)..${ }^{14-16}$ Recently, the high-resolution imaging techniques, such as 2D-3D ultrasonography and magnetic resonance imaging (MRI), have also been applied in
Correspondence: Gloria Calagna, Obstetrics and Gynecology Unit, "Villa Sofia Cervello" Hospital, University of Palermo, via Trabucco 180, 90145 Palermo, Italy.

Tel.: 091.6802194 - Fax: 091.6802176.

E-mail: gloria.calagna83@gmail.com

Key words: Urethral diverticulum; Diagnostic tool; Female; Diagnosis.

Contributions: GCa: conception of the article, interpretation of data, drafting the article; MV: interpretation of data, revising article critically for important intellectual content; MM, GG: analysis of data, drafting the article; SP, GCu: design of the article, interpretation of data; AP: revising article critically for important intellectual content. All authors gave final approval of the version to be published.

Conflict of interest: the authors declare no potential conflict of interest.

Funding: none.

Received for publication: 14 June 2018

Revision received: 26 November 2018.

Accepted for publication: 21 March 2019.

This work is licensed under a Creative Commons Attribution NonCommercial 4.0 License (CC BY-NC 4.0).

CC Copyright G. Calagna et al., 2019

Licensee PAGEPress, Italy

Urogynaecologia 2019; 31:222

doi:10.4081/uij.2019.222

the study of the abnormalities of the female urethra, with high sensitivity and less invasiveness. Here, we report and comment the available tools in the diagnosis of UD, focusing particularly on the current role of VCUG.

\section{Voiding cystourethrography}

VCUG is the oldest and most traditional instrument to diagnose female UD, considered as the imaging modality of choice providing data regarding the number, location, and size of the UD as well as communication with the urethra. ${ }^{17}$ In the past, many authors supported the efficiency of this approach: Ganabathi et al. in 1994 demonstrated the presence of UD in $95.2 \%$ of a large series of 63 women using VCUG. ${ }^{18}$

The VCUG procedure is technically easy and simple. ${ }^{19}$ With the patient in the standing position and using a 14-F Foley catheter, the operator fills the bladder with contrast agent. Although 15\% dilution of 
ionic contrast medium diatrizoato meglumine produces good images, a non-ionic contrast medium (Omnipaque ${ }^{\mathrm{TM}}$ or Ultravist) is generally preferred.

Technically, it is important to achieve the anteroposterior and oblique images of bladder and urethra during filling as well as during voiding and after voiding: when UD is present, the images obtained during (Figure 1) or after voiding (Figure 2) can generally demonstrate total or partial filling of the diverticular sac. Moreover, filling defects within diverticula may suggest the possibility of urethral calculi or tumors. ${ }^{7}$ The voiding phase of intravenous urography, although useful for the diagnosis of duplex system with ectopic ureterocele, is nowadays performed with ultrasound, CT and MRI studies. However, VCUG has some known limits: the procedure is invasive, and time consuming, the patient is exposed to ionizing radiations and requires catheterization. ${ }^{19}$ Furthermore, successive and more recent experiences reported far less favorable UD detection rates, questioning the VCUG role as principal diagnostic tool. ${ }^{20,21}$ In particular, low accuracy in the definition of the precise localization of the diverticular orifice was highlighted, as well as in the correct identification of number and size of UD, mainly when the UD is only partially opacified. ${ }^{22}$ In 2003, Golomb et al. published the results of a comparison between the information obtained by VCUG and positive-pressure DBU on 12 women with a presumptive clinical diagnosis of UD, in order to verify which imaging modality can better delineate the features of the diverticula. ${ }^{19}$ In 4 out of 12 patients (33.3\%), VCUG completely failed in showing the diverticulum, whereas DBU showed a large complex diverticulum in 2 patients and a distinct mid-urethral diverticulum in 2 patients; in the remaining 8 women (66.7\%), VCUG delineated only the lower part of the diverticulum, whereas DBU depicted a large diverticulum extending beneath the bladder neck in 3 patients and multiple diverticula in 5 patients. The sensitivity of DBU and VCUG, was defined therefore at 100 and $66.7 \%$, respectively. ${ }^{19}$

Based on the evidence of these recent comparative reports, VCUG results have been equivocal or non-confirmatory, and often needed additional imaging studies; in this sense, and considering the availability of other effective diagnostic options, the VCUG could be used mainly as a screening test, and if it fails to provide adequate characterization of the UD, one of the other imaging modalities could be applied. ${ }^{17}$ Finally, VCUG may be a useful tool for the contemporary diagnosis of an eventual vesical-urethral reflux - a possible cause of per- sistent urinary infection like UD - and, when it is performed during a video-urodynamic investigation, in order to evaluate bladder dysfunction such as incontinence, hyperactivity and obstruction, which are often the cause of symptoms, and the underlying mechanism of UD genesis.

\section{Double-balloon urethrography}

The first DBU was performed and reported in $1959 .{ }^{23}$ DBU is a positive pressure retrograde urethrography and to perform this procedure, it is necessary to use a specialized 14-F Foley catheter with a second balloon or a retention plug proximal to the balloon at the catheter tip: the passive bladder filling with contrast agent is obtained at a higher pressure.

In particular, the bladder balloon is filled to $20 \mathrm{ml}$ to close the bladder neck; the second balloon, running to the catheter, is placed close to the external urethral meatus; the catheter between the balloons is open and, in this way, the contrast medium can be injected into a closed camera, realizing a positive pressure urethrography. It is important that the operator ties the balloons in the right position during the examination. ${ }^{19}$

In comparison with VCUG, the DBU technique has an extra weapon as it works under positive pressure. The positive pressure, in fact, allows to force contrast agent into a diverticular orifice during the injec- tion and can be identified at early phase of filling of the UD. ${ }^{24}$ In this sense, the reason why VCUG fails in some patients while DBU is successful may be due to a narrow communication of the diverticulum to the urethra, which, during normal voiding, blocks the viscous contrast medium from entering the diverticulum, whereas the positive-pressure created during DBU overcomes the resistance and enables proper visualization of the diverticulum. ${ }^{19}$

Although DBU has proved to be more sensitive than traditional VCUG as a diagnostic test for female UD, ${ }^{19,24}$ it has several limitations. ${ }^{24,25}$ It is technically more challenging to perform than VCUG, with the technical difficulty of creating an ideal closed urethral system by the catheter; the procedure may be painful for the patient and exposes the patient and operator to ionizing radiation. ${ }^{26,27}$ Moreover, increased risk of urinary tract infection and/or urethral injury have led to a drop in the usage of this procedure. ${ }^{27}$

\section{Ultrasonography}

Transabdominal, transrectal and transperineal techniques have been described in the last few years for the assessment of UD, but despite their noninvasiveness, these approaches are not widely diffused in routine practice, mainly because of the insensitivity for detecting

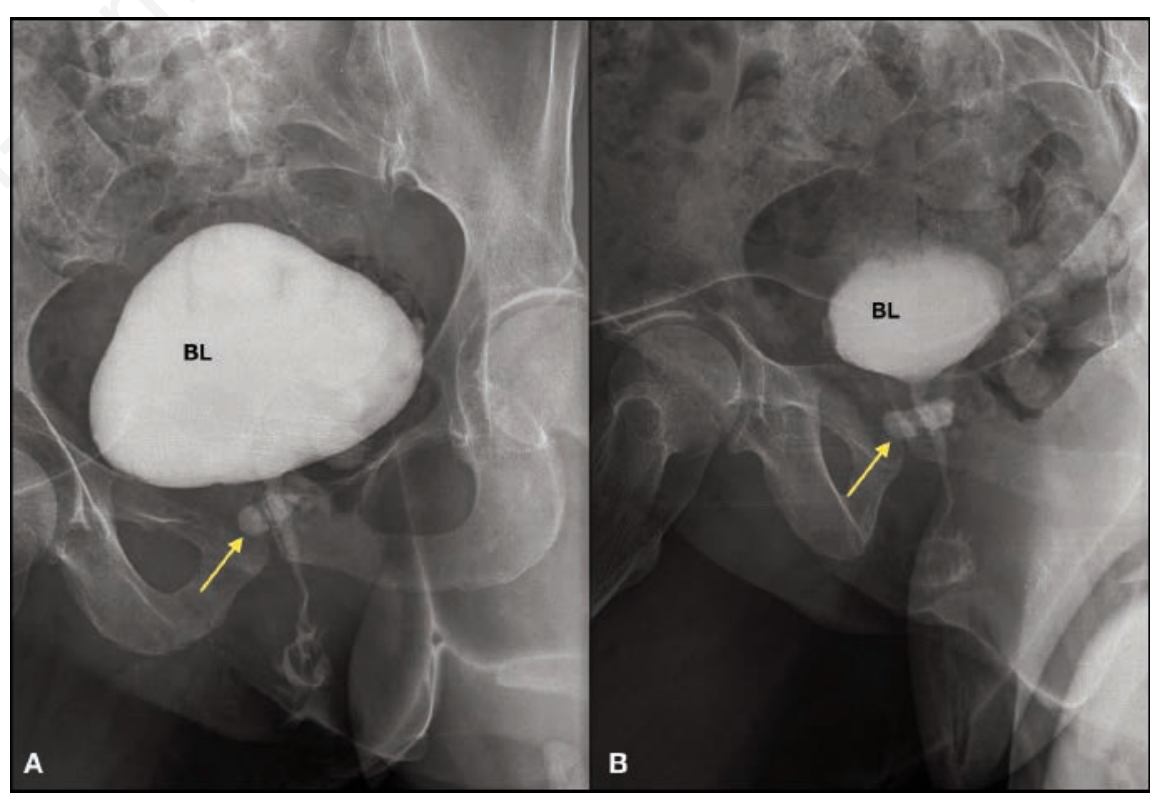

Figure 1. A 52-year-old woman with recurrent urinary tract infections. On physical examination a periurethral mass was present with expression of pus from the urethra. (A) VCUM showed a diverticulum in the middle urethra; (B) the bladder is almost completely voided and the diverticulum is seen surrounding the urethra in a horseshoe shape (BL: bladder; yellow arrow: urethral diverticulum). 
small diverticula $(<2 \mathrm{~cm}){ }^{10,28}$

Transvaginal US (TV-US) $(7-10 \mathrm{MHz})$ has also been tried for the study of urethra and abnormalities, with better results, being able to detect UD that do not fill with contrast agent. With this approach, the urethra is fully visible from the meatus to the bladder neck in different planes, and additional information on UD characteristics (size, number, site, content) may be achieved. ${ }^{29}$ The advantages of US include absence of ionizing radiation, wide availability, ability to differentiate the type of mass (solid $v s$ cystic) and the precise relationship of the diverticulum to the urethra; finally, it is the imaging method of choice for pregnant patients with suspicion of urethral pathology. However, TV-US has the relevant disadvantage of involving direct urethral compression as well as poor resolution for deep soft tissue, inter-operator variability, and differentiating UD from other peri-urethral cystic diseases. . $^{5,10}$

Referring to US techniques applied to urogynecology, a new entry is the translabial ultrasound (TL-US) approach, which has proved to be a valid option in the study of disorders of the lower urogenital tract. ${ }^{30}$ To perform a correct TL-US evaluation, the principal procedural phases are the following: i) probe placement on the anterior vulvar commissure (directed to the anterior vaginal wall), firstly on the median sagittal plane under the pubis; ii) initial image acquisition (sagittal plane), showing the arcuate ligament of the pubis, the bladder with bladder neck and the longitudinal section of the urethra; the arcuate ligament of the pubis appears as a hyperechoic image and is a crucial landmark; the urethral lumen appears as a transonic channel bounded by hypoechoic outline; iii) image acquisition on the coronal plane (rotating the probe $90^{\circ}$ anti-clockwise), focusing on the urethral lumen and any eventual abnormalities $^{5}$ (Figure 3).

The periurethral mass may be easily detected and its echogenicity evaluated: a neoformation is defined as UD only if a communication channel to the urethral lumen is identifiable. In 2009, El-Zein et al. reported their experience in locating the UD neck intra-operatively, in patients in which cystoscopy had failed: they showed the relevant role of TL-US as a diagnostic tool and as surgical guidance, identifying $95 \%$ of the UD without the help of level II diagnostic methods. ${ }^{31}$ Then, Gugliotta et al., in 2015, suggested the use of TL-US as a first-line method for the evaluation of urethral masses in an outpatient setting, especially when UD is clinically suspected, also focusing on the sonographic characteristics in differential diagnosis with the para-urethral and

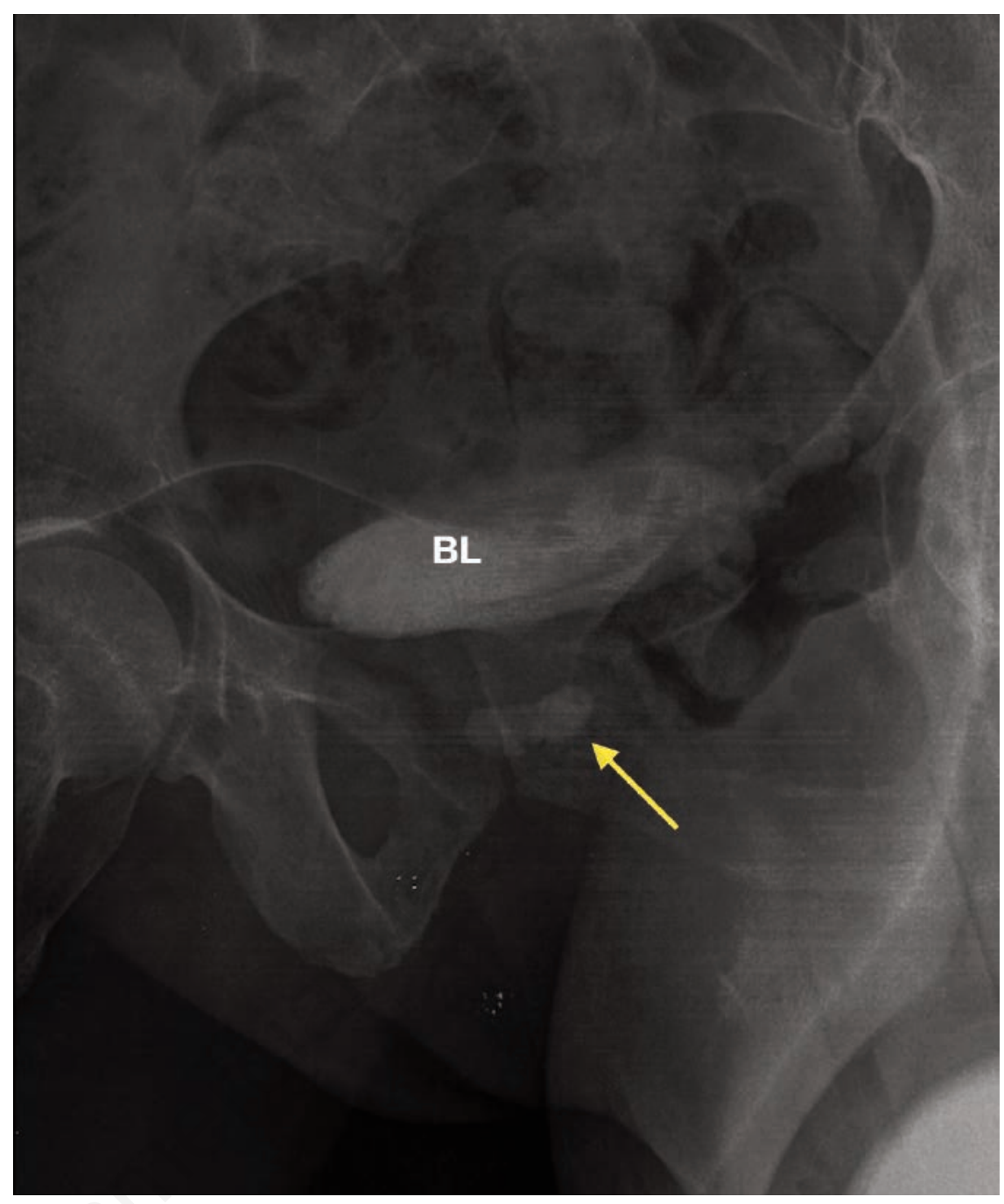

Figure 2. The diverticulum is clearly seen in after voiding film (BL: bladder; yellow arrow: urethral diverticulum).

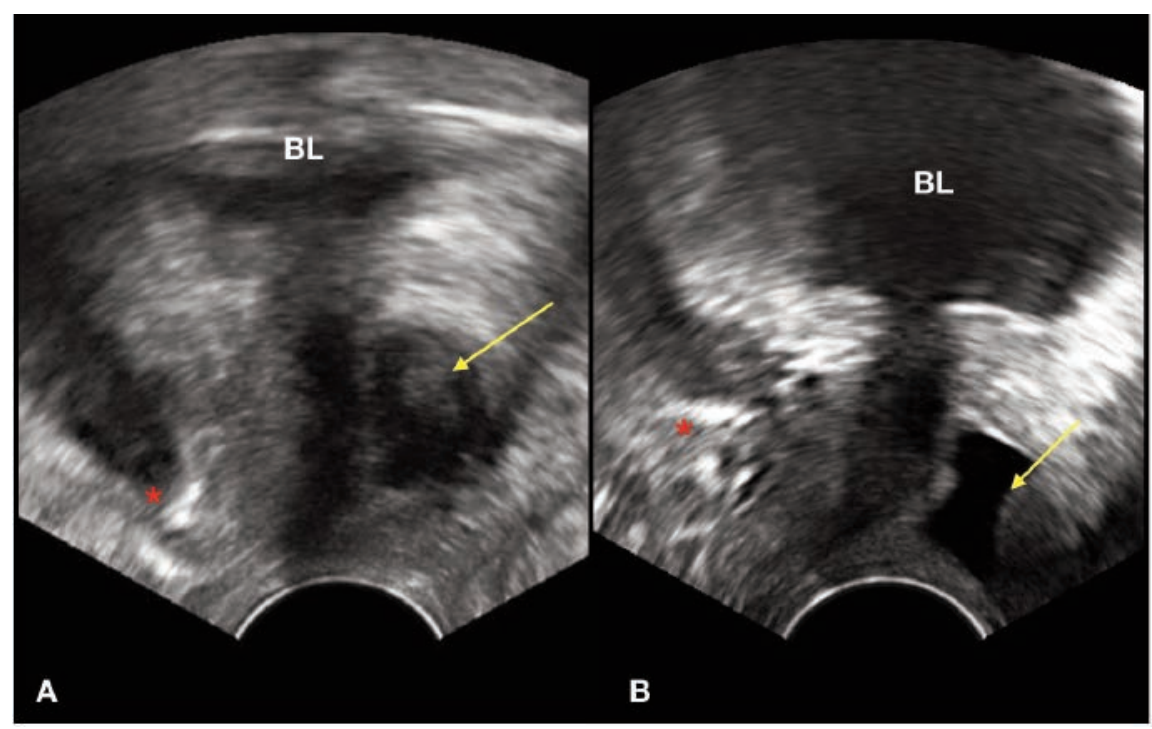

Figure 3. Trans-labial ultrasound of a simple (A) and complex (B) urethral diverticulum in longitudinal section (BL: bladder; yellow arrow: urethral diverticulum; red star: pubis) 
vaginal cyst. ${ }^{5}$ In addition to minimal invasiveness, TL-US allows to achieve the images from various angles of view, evaluating the spatial relationship of the UD to the urethra. Certainly, a skilled operator is necessary.

\section{Magnetic Resonance Imaging}

MRI plays an important role in the evaluation of the urogenital tract and, in particular, urethral diseases in women. Literature is not extensive but other groups have reported almost $100 \%$ MRI sensitivity to diagnose UD. ${ }^{32-34}$

It may be performed with a torso phased-array coil or an endoluminal (endorectal, endovaginal, or endourethral) coil, thereby allowing improved signal-tonoise ratio and high-resolution imaging. ${ }^{35,36}$ Female urethral examinations are performed on a 1.5-T MR imager with a pelvic phased-array coil. The suggested MRI protocol consists of axial, coronal, and sagittal fat-saturated fast spin echo T2-weighted sequences (repetition time msec/echo time msec $=4,000-5,500 / 80-120$, three signals acquired, echo train length of 12, 18-24-cm field of view, 3-5-mm section thickness, no intersection gap, $512 \times 512$ matrix).?

The intravenous administration of contrast medium can be helpful in differentiating a condition of inflammation from malignancy in the context of a UD; possible malignancy arising from a diverticulum can be visualized as enhancing soft tissue within the diverticulum. On the other hand, endoluminal MRI is excellent for defining the characteristics of a lesion that involve the urethra: it has been able to confirm the presence of the orifice of the UD or assess the non-communication of the urethral mass. ${ }^{37}$ This approach allows good recognition of the urethral diverticular cavity: single or multiple, unilocular or multilocular, internal acute inflammation or neoplasm (mainly after the administration of contrast agent); moreover, considering the soft-tissue contrast capacity, MRI adequately differentiates a solid mass from a complex diverticulum with septa (Figure 4).

The advantages of MRI include multiplanar capability, with an optimal characterization of female urethral and periurethral diseases or its abnormalities, ${ }^{38}$ and lack of ionizing radiation. However, the main disadvantages include high cost and longer examination time. ${ }^{13}$ It is worth noting that more recent experiences on the topic with the addition of surgical data seem to limit the paradigm that MRI is the gold standard to diagnose UD. Kim et al. evaluated the role of MRI in the diagnosis of UD in 20 patients showing sensitivity limited to $70 \%$ but with higher accuracy than VCUG and UC: 14 vs 11 out of 20 diverticula, respectively identified with MRI and VCUG/UC. Authors, however, highlighted that the use of MRI contrast medium is able to enhance the characteristics of the content of the UD, such as in showing granulation tissue or carcinoma. ${ }^{39}$ Chung et al., in 2010, reported a discrepancy between MRI and surgical findings in a sample of 76 patients who underwent diverticulectomy: in 41 patients who had previously undergone MRI, 10 (24.4\%) were diagnostic errors (diverticula were not seen on MRI in 3 cases). ${ }^{32}$ To explain these diagnostic errors, authors proposed different hypotheses; UD did not appear fluid filled on T2-weighted imaging and it is dynamic by nature with constant fluctuation in size. In this sense, a limitation of MRI is that it captures images at a single point in time, in contrast to more dynamic tests that capture real-time images, such as VCUG. ${ }^{32}$ Some recent studies also show that MRI does not have excellent sensitivity in detecting ostia. ${ }^{32,33,40}$ However, MRI is certainly a relevant tool to evaluate UD, especially in the pre-operative phase, but physicians should be aware of its limitations.

\section{New tools: computer tomogra- phy-voiding cystourethrography}

Recent evolutions in multidetector CT have made $2 \mathrm{D}$ and $3 \mathrm{D}$ reformatted $\mathrm{CT}$ images available for several diagnostic fields. ${ }^{7}$ The new-generation CT scanners (with faster scanning speeds) allowed urethral evaluation with new techniques as CT voiding cystourethrography (CT-VCUG) and virtual urethroscopy. In this field, the use of a 16-MDCT scanner is innovative, with a detector configuration of 0.75 $\mathrm{mm} \sim 16$ and a pitch of 1.25 . The gantry is firstly positioned at the level from which the scanning starts (the top of the bladder) and then the patient is asked to void. When this signal is noticed, an unenhanced scan down to the inferior margin of symphysis pubis is achieved. ${ }^{21}$ The scanning time is very fast (about 7 seconds). Axial images are reconstructed with a 1-mm thickness and interval; thin-slab (2-mm) coronal and sagittal images and 3D CT urethrographic images, as well as CT virtual urethroscopic images, are reformatted..$^{21}$

The multidetector CT has several advantages in comparison with standard techniques: rapid scanning, thin collima-

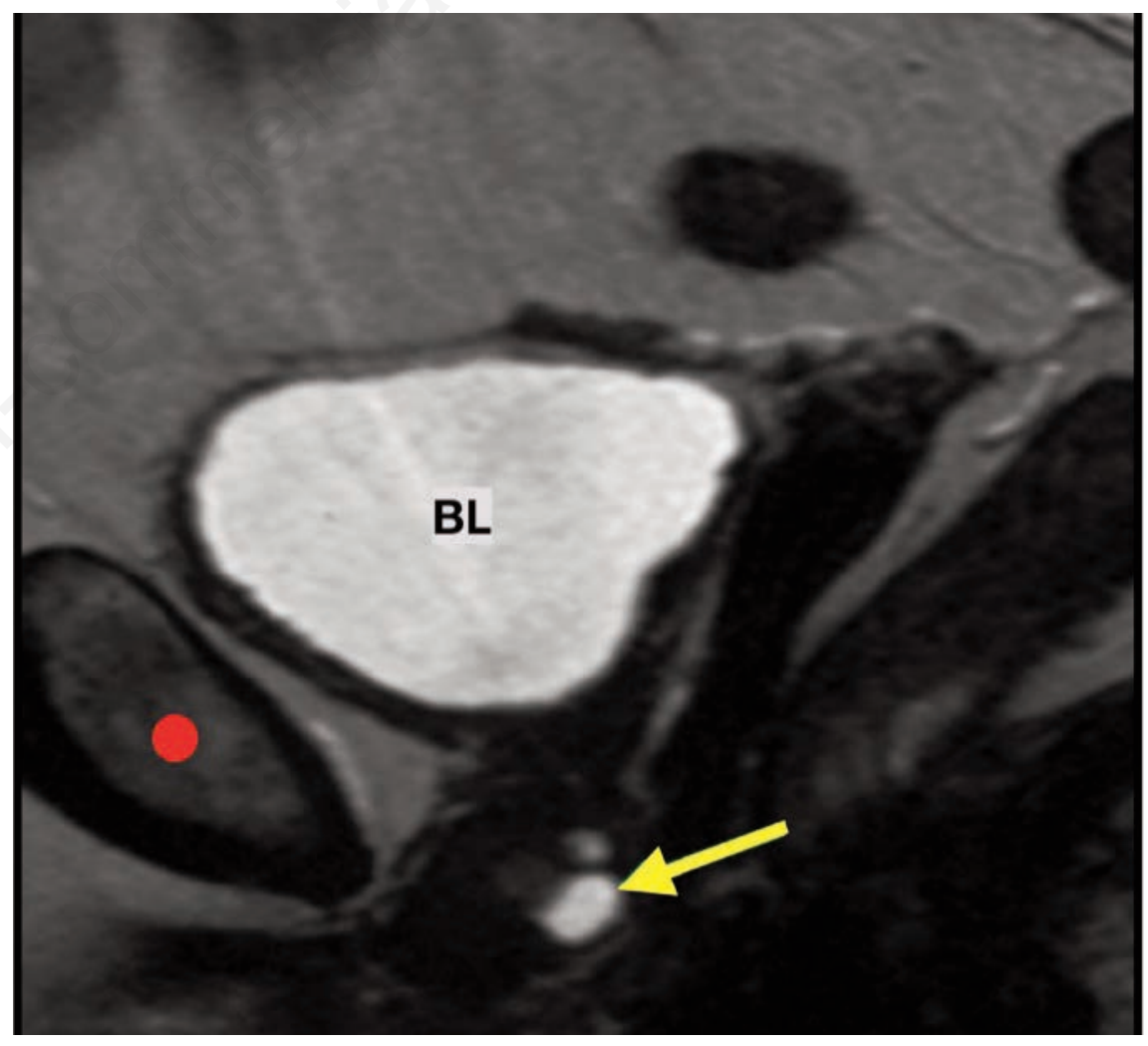

Figure 4. Magnetic resonance imaging (with contrast medium) of female urethral diverticulum (BL: bladder; yellow arrow: urethral diverticulum; red circle: pubis). Reproduced with permission from: Gugliotta G, Calagna G, Adile G, et al. Use of trans-labial ultrasound in the diagnosis of female urethral diverticula: A diagnostic option to be strongly considered. The Journal of Obstetrics and Gynaecology Research 2015;41:1108-14. doi:10.1111/jog.12676 
tion, and highly improved resolution of the z-axis; these technical characteristics allow the multiplanar and 3D reformatted images of high quality, comparable to those of conventional VCUG. ${ }^{7}$

This approach can show the accurate measurement of diverticulum size, the simultaneous viewing of 2D and 3D images of the urethra (without magnification or distortion) and the presence and exact location of the opening of the UD in the urethral lumen, and clearly visualize the neck; this last capacity makes the multidetector CT unique in this diagnostic field, as identification of the neck of the UD is almost impossible with other imaging techniques. ${ }^{41}$ Moreover, it is associated to good patient compliance, minimal organ injury and less discomfort compared with traditional UC. Lee et al., in 2014, described the clinical usefulness of CT-VCUG using a 16- multidetector $\mathrm{CT}$ in pre-operative evaluation of female UD. In this study, authors reported an exceptional result on 14 consecutive patients who underwent urethral diverticulectomy and were previously studied using the CT-VCUG technique: diverticular ostium was detected by CT-VCUG in all the considered cases, while only in $71.4 \%$ by UC. ${ }^{41}$ However, some difficulties still need to be overcome to achieve real diffusion in the practice of this imaging technique. In fact, the procedure is time-consuming and voiding while lying on the CT table may be not easy for some patients, thereby lengthening the timing of exam or making the evaluation impossible to obtain adequate voiding images; ${ }^{21}$ nevertheless, this last limitation is identical for conventional VCUG. Finally, the problem of radiation should be considered for the patients in reproductive age (the genitalia are within the scan regions).

\section{Recent findings}

In the last years, the international literature focused different aspects of the topic. First of all, more attention has been placed on the diagnostic aspects of videourodynamics (VUDS), known to be useful in 62$95 \%$ of patients with UD and also providing additional information on associated urinary disfunction. ${ }^{42}$ In a retrospective case note review of 20 women with symptomatic UD, VUDS was diagnostic in 90\% $(n=18)$ of UD patients, being helpful in accurately characterizing the symptoms. In $15 \%$ of cases, stress urinary incontinence was associated; high detrusor pressure at maximum flow rate and low catheter-free uroflow rate, which indicated the possibilities of bladder outlet obstruction, were observed $(40 \%$ of cases). ${ }^{42}$ The relevant role of TLUS was confirmed by data from a large retrospective study on 4121 women, examined with 3D/4D TLUS and urethroscopy with a 0 degree cystoscope. ${ }^{43}$ In 25 cases $(0.6 \%)$ were found a major urethral abnormality on TLUS and in 17 case the cystic structure it has been hypothesized to be an UD: urethroscopy confirmed the diverticulum in 16 cases (94\%). ${ }^{43}$ In 2017, Zhao et al. published data on the use of the 640-Multislice CT (640-MSCT), with 3D and 4D reconstruction, to ascertain the existence of the UDs and to figure out their locations, sizes, ostia and shapes in 16 female patients. ${ }^{44}$ Using new-generation 640-MSCT, images of a contrast agent-filled urethra during patient voiding were obtained in approximately 5 seconds, providing more detailed urethral structure, which made it possible to identify the UD ostia easily and clearly; also, the high resolution of the system made it feasible to detect the small amount of contrasts passing through the ostia, even the narrow ones. Finally, with the help of post processing techniques, authors reformatted $3 \mathrm{D}$ and $4 \mathrm{D}$ images, and all patients were diagnosed accurately pre-operation, with a positive predictive values of $100 \%$ in diagnosis of female UD. ${ }^{44}$ Highly interesting was the recent rare case of a large UD complicating pregnancy in third trimester, influencing the mode of delivery, performed by cesarean section. ${ }^{45}$ In fact, the diagnosis and management of UD in pregnancy are challenging because of the rare nature of the condition, the varied presentations and the possibility of misdiagnosis, often related to absence of pelvic examination during second and third trimester. However, early identification of UD during pregnancy may allow for possible aspiration and trial of labor with the anticipation of a vaginal delivery. A notable review of English and Japanese literature on the problem of urethral diverticulum carcinoma (UDC) in women, an extremely rare condition (only 126 reported cases) with several diagnostic difficulties because of its nonspecific presentation. ${ }^{46}$ Urine cytology may be a useful initial screening test and has been reported to be positive in $10 / 11(91 \%)$ cases in which it was utilised. The gold standard investigations to date seems to be a combination of gadolinium-enhanced MRI which can be used for diagnosis, staging and surveillance followed by transvaginal trucut or transurethral biopsies for definitive diagnosis. Moreover, cystoscopy may play an important role in the pathological diagnosis and in localization of the tumor origin and CT may be used to assess for lymph node enlargement, distant tissue and bone metastasis. ${ }^{46}$

\section{Conclusions}

UD is still difficult to diagnose and certainly, the first essential step for a timely diagnosis is the idea of the possibility of a UD in women with persistent lower urinary tract symptoms. Clinicians should know the main imaging features of this pathology and also the multiple, currently available techniques for its correct diagnosis. The key point of successful surgical management of UD is correct identification of the ostium.

Current literature data confirm a resized role of conventional VCUG alone in the diagnosis of UD. It is not always sufficient for the detection of diverticula and the ostia, and is considered a highly invasive technique. However, it should be remembered that video-urodynamic could be useful in the case of coexistent functional abnormalities, such as incontinence or obstruction. A similar comment can be made for DBU, which has been introduced to improve sensitivity, but the question of invasiveness remains. TL-US is the new favored tool thanks to its minimal invasiveness and easy retrieval, but it still has poor specificity in differentiation from other peri-urethral lesions; consequently, implementation with UC is often needed. It is relevant to also consider that UC has many limitations (such as the definition of diverticular mass features), and moreover it sometimes fails, especially in the difficult case when infection or obstruction is present in the neck of a diverticulum.

Today, MRI and CT-VCUG appear to be the most efficient techniques in the diagnosis and pre-operative definition of female UD. MRI is superior to other techniques in its sensitivity and in showing the relationship between UD and urethral canal, thanks to the multiplane scan and good soft-tissue contrast, but often it does not show the exact location of the ostium. The innovation of 3D-MR sequences and the endorectal coil have improved the traditional results of MRI: the long scanning time has been reduced and the resolution increased. Certainly, the high cost of MRI and its technical accessories remain the main limitation and not all centers have MRI availability.

The use of CT-VCUG (and virtual urethroscopy), while having the classic disadvantage of the conventional contrast enhanced radiologic procedures, represent a very reliable approach providing structural information of the UD, giving both urethral and extraluminal high quality anatomic information. Moreover, it is associated to good patient compliance and minimal organ injury. However, the priority element in these rare cases of UD is above all to suspect the possibility of the pathology in the presence of 
significant lower urinary tract symptoms in a woman, in order to get a prompt diagnosis.

\section{References}

1. Hey W. Practical observations in surgery. Philadelphia: James Humphreys; 1805. p 303.

2. Singla P, Long SS, Long CM, et al. Imaging of the female urethral diverticulum. Clin Radiol 2013;68:e418-25.

3. El-Nashar SA, Bacon MM, Kim-Fine S, et al. Incidence of female urethral diverticulum: a population-based analysis and literature review. Int Urogynecol J 2014;25:73-9.

4. Ljungqvist L, Peeker R, Fall M. Female urethral diverticulum: 26-year followup of a large series. J Urol 2007;177:21924.

5. Gugliotta G, Calagna G, Adile G, et al. Use of trans-labial ultrasound in the diagnosis of female urethral diverticula: A diagnostic option to be strongly considered. J Obstet Gynaecol Res 2015; 41:1108-14

6. Ingber MS, Firoozi F, Vasavada SP, et al. Surgically corrected urethral diverticula: Long-term voiding dysfunction and reoperation rates. Urology 2011;77:65-9.

7. Chou CP, Levenson RB, Elsayes KM, et al. Imaging of female urethral diverticulum: An update. Radiographics 2008;28:1917-30.

8. Leach GE, Bavendam TG. Female urethral diverticula. Urology 1987;30:40715.

9. Lee TG, Keller FS. Urethral diverticulum: Diagnosis by ultrasound. AJR Am J Roentgenol 1977;128:690-1.

10. Keefe B, Warshauer DM, Tucker MS, Mittelstaedt CA. Diverticula of the female urethra: Diagnosis by endovaginal and transperineal sonography. AJR Am J Roentgenol 1991;156:1195-7.

11. Siegel CL, Middleton WD, Teefey SA, et al. Sonography of the female urethra. AJR Am J Roentgenol 1998;170:126974.

12. Arunkalaivanan AS, Baptiste M, Sami $\mathrm{T}$. Urethral diverticulum in women: retrospective case series. J Obstet Gynecol India 2016;66:47-51.

13. Antosh DD, Gutman RE. Diagnosis and managment of female urethral diverticulum. Female Pelvic Med Reconstr Surg 2011;17:264-71.

14. Boyd SD, Raz S. Female urethral diverticula. In: Raz S, ed. Female Urology. Philadelphia: Saunders; 1983. pp 378396.

15. Peters WA, Vaughan ED. Urethral diverticulum in the female. Etiologic factors and postoperative results. Obstet Gynecol 1976;47:549-52.

16. Stewart M, Bretland PM, Stidolph NE. Urethral diverticula in the adult female. Br J Urol 1981;53:353-9.

17. Wang AC, Wang CR. Radiologic diagnosis and surgical treatment of urethral diverticulum in women. A reappraisal of voiding cystourethrography and positive pressure urethrography. J Reprod Med 2000;45:377-82.

18. Ganabathi K, Leach GE, Zimmern PE, Dmochowski R. Experience with management of urethral diverticulum in 63 women. J Urol 1994;152:1445-52.

19. Golomb J, Leibovitch I, Mor Y, et al. Comparison of voiding cystourethrography and double-balloon urethrography in the diagnosis of complex female urethral diverticula. Eur Radiol 2003;13:536-42.

20. Romanzi LJ, Groutz A, Blaivas, JG. Urethral diverticulum in women: diverse presentations resulting in diagnostic delay and mismanagement. J Urol 2000;164:428-33.

21. Kim SH, Kim SH, Park BK, et al. CT voiding cystourethrography using 16MDCT for the evaluation of female urethral diverticula: initial experience. AJR Am J Roentgenol 2005;184:1594-6.

22. Khati NJ, Javitt MC, Schwartz AM, Berger BM. MR imaging diagnosis of a urethral diverticulum. Radiographics 1998;18:517-22.

23. Drutz HP. Urethral diverticula. Obstet Gynecol Clin North Am 1989;16:923-9.

24. Jacoby K, Rowbotham RK. Double balloon positive pressure urethrography is a more sensitive test than voiding cystourethrography for diagnosing urethral diverticulum in women. J Urol 1999; 162:2066-9.

25. Handel LN, Leach GE. Current evaluation and management of female urethral diverticula. Curr Urol Rep 2008;9:3838.

26. Lee RA. Diverticulum of the urethra: Clinical presentation, diagnosis and management. Clin Obstet Gynecol 1984;27:490-8.

27. Neitlich JD, Harris Jr E, Glickman MG, Smith RC. Detection of urethral diverticula in women: comparison of a high resolution fast spin echo technique with double balloon urethrography. J Urol 1998;159:408-10.

28. Wexler JS, McGovern TP. Ultrasonography of female urethral diverticula. AJR Am J Roentgenol 1980;134:737-40.

29. Fontana D, Porpiglia F, Morra I, Destefanis P. Transvaginal ultrasonog- raphy in the assessment of organic diseases of female urethra. J Ultrasound Med 1999;18:237-41.

30. Martensson O, Duchek M. Translabial ultrasonography with pulsed colourDoppler in the diagnosis of female urethral diverticula. Scand J Urol Nephrol 1994;28:101-4.

31. El-Zein C, Khoury N, El-Zein Y, et al. Intraoperative translabial ultrasound for urethral diverticula: A road map for surgeons. Eur J Radiol 2009;70:133-7.

32. Chung DE, Purohit RS, Girshman J, Blaivas JG. Urethral diverticula in women: Discrepancies between magnetic resonance imaging and surgical findings. J Urol 2010;183:2265-9.

33. Ockrim J, Allen D, Shah P, et al. A tertiary experience of urethral diverticulectomy: diagnosis, imaging and surgical outcomes. BJU Int 2009;103:15504.

34. Foster R, Amundsen C, Webster G. The utility of magnetic resonance imaging for diagnosis and surgical planning before transvaginal periurethral diverticulectomy in women. Int Urogynecol J Pelvic Floor Dysfunct 2007;18:315-9.

35. Macura KJ, Genadry R, Borman TL, et al. Evaluation of the female urethra with intraurethral magnetic resonance imaging. J Magn Reson Imaging 2004;20: 153-9.

36. Elsayes KM, Mukundan G, Narra VR, et al. Endovaginal magnetic resonance imaging of the female urethra. J Comput Assist Tomogr 2006;30:1-6.

37. Daneshgari F, Zimmern PE, Jacomides L. Magnetic resonance imaging detection of symptomatic noncommunicating intraurethral wall diverticula in women. J Urol 1999;161:1259-62.

38. Siegelman ES, Banner MP, Ramchandani P, Schnall MD. Multicoil MR imaging of symptomatic female urethral and periurethral disease. RadioGraphics 1997;17:349-65.

39. Kim B, Hricak H, Tanagho EA. Diagnosis of urethral diverticula in women: Value of MR Imaging. AJR Am J Roentgenol 1993;161:809-15.

40. Portnoy O, Kitrey N, Eshed I, et al. Correlation between MRI and doubleballoon urethrography findings in the diagnosis of female periurethral lesions. Eur J Radiol 2013;82:2183-8.

41. Lee YJ, Son SJ, Paick JS, Kim SW. Preoperative CT voiding cystourethrography using 16-multidetector $\mathrm{CT}$ in female urethral diverticulum. PLoS One 2014;9:e107448.

42. Kuan-Jung L, Yu-Hua F, Alex TongLong L. Role of urodynamics in management of urethral diverticulum in 
females. J Chin Med Assoc 2017;80:712e716.

43. Guichard P, Gillor M, Caudwell-Hall J, Dietz HP. OP20. 03: Translabial ultrasound for the diagnosis of urethral diverticula. Ultrasound Obstet Gynecol 2018;52:126-6.

44. Zhao YX, Wang JP, Li JM, et al.
Preoperative 3D and 4D-CT imaging using 640-Multislice CT (640-MSCT) in diagnosis of female urethral diverticulum. World J Urol 2017;35:1133-9.

45. Magann EF, Newton LS, Barr SA. Urethral diverticulum presenting as a large vaginal mass complicating pregnancy and delivery. Am J Case Rep
2017; 18:1095-8.

46. O'Connor E, Iatropoulou D, Hashimoto $\mathrm{S}$, et al. Urethral diverticulum carcinoma in females - a case series and review of the English and Japanese literature. Transl Androl Urol 2018;7:703-29. 